\title{
Three-Dimensional Intracellular Calcium Gradients in Single Human Burst-forming Units-Erythroid-derived Erythroblasts Induced by Erythropoietin
}

Rao V. Yelamarty, Barbara A. Miller, Russell C. Scaduto, Jr., Francis T. S. Yu, Douglas L. Tillotson, and Joseph Y. Cheung Departments of Medicine, Pediatrics, Physiology and Surgery, The Milton S. Hershey Medical Center, The Pennsylvania State University, Hershey, Pennsylvania 17033; Department of Electrical Engineering, The Pennsylvania State University, University Park, Pennsylvania 16802; and Department of Physiology, Boston University School of Medicine, Boston, Massachusetts 02118

\begin{abstract}
We have previously shown that the intracellular free $\mathrm{Ca}^{2+}$ increase induced by erythropoietin is likely related to differentiation rather than proliferation in human BFU-E-derived erythroblasts (1989. Blood. 73:1188-1194.). Since cell differentiation involves transcription of specific regions of the genome, and since nuclear endonucleases responsible for single strand DNA breaks observed in cells undergoing differentiation are $\mathrm{Ca}^{2+}$ dependent, we investigated whether the erythropoietin-induced calcium signal is transmitted from cytosol to nucleus in this study. To elucidate subcellular $\mathrm{Ca}^{2+}$ gradients, the technique of optical sectioning microscopy was used. After determining the empirical three-dimensional point spread function of the video imaging system, contaminating light signals from optical planes above and below the focal plane of interest were removed by deconvolution using the nearest neighboring approach. Processed images did not reveal any discernible subcellular $\mathrm{Ca}^{2+}$ gradients in unstimulated erythroblasts. By contrast, with erythropoietin stimulation, there was a two- to threefold higher $\mathrm{Ca}^{2+}$ concentration in the nucleus compared to the surrounding cytoplasm. We suggest that the rise in nuclear $\mathrm{Ca}^{2+}$ may activate $\mathrm{Ca}^{2+}$-dependent endonucleases and initiate differentiation. The approach described here offers the opportunity to follow subcellular $\mathrm{Ca}^{2+}$ changes in response to a wide range of stimuli, allowing new insights into the role of regional $\mathrm{Ca}^{2+}$ changes in regulation of cell function. (J. Clin. Invest. 1990. 85:1799-1809.) fura-2 • nuclear calcium - cell differentiation • optical sectioning microscopy • subcellular calcium heterogeneity
\end{abstract}

\section{Introduction}

The intracellular free calcium concentration $\left[\mathrm{Ca}_{\mathrm{i}}\right]$ has been shown to be an intracellular signal for many hormones (1) and growth factors (2). We have previously demonstrated that erythropoietin (Epo $)^{1}$ increased $\left[\mathrm{Ca}_{\mathrm{i}}\right]$ in single early human

Address reprint requests to Dr. Cheung, Division of Nephrology, Department of Medicine, The Milton S. Hershey Medical Center, P. O. Box 850, Hershey, PA 17033.

Received for publication 7 August 1989 and in revised form 6 February 1990

1. Abbreviations used in this paper: $\mathrm{CCD}$, charge coupled device; Epo, erythropoietin; IMDM, Iscove's modified Dulbecco's medium; NA, numerical aperture; PSF, point spread function; 3-D, three-dimensional; 2-D, two-dimensional.

J. Clin. Invest.

(c) The American Society for Clinical Investigation, Inc.

$0021-9738 / 90 / 06 / 1799 / 11 \quad \$ 2.00$

Volume 85, June 1990, 1799-1809 erythroid precursors (3). Subsequent studies suggested that Epo-induced increase in $\left[\mathrm{Ca}_{\mathrm{i}}\right]$ may be related to promotion of cell differentiation rather than proliferation since Epo-induced elevations in $\left[\mathrm{Ca}_{i}\right]$ were observed in later erythroid precursors but not in early precursors $(4,5)$.

The molecular mechanisms by which growth factors initiate the cascade of transmembranous and intracellular events leading to gene transcription are as yet poorly understood. Since cell differentiation ultimately involves transcription of selected regions of the genome, it is important to investigate whether the $\mathrm{Ca}^{2+}$ signal observed in human erythroid precursors stimulated with Epo is transmitted to the nucleus. There are several known intranuclear enzymes that are sensitive to $\mathrm{Ca}^{2+}$ or calmodulin in vitro (6-8). Demonstration of nuclear $\mathrm{Ca}^{2+}$ signaling by growth factors will help focus on the physiologic relevance of $\mathrm{Ca}^{2+}$-sensitive intranuclear enzymes on gene transcription.

In the present study, nuclear free $\mathrm{Ca}^{2+}$ concentration $\left[\mathrm{Ca}_{n}\right]$ in day 10 single BFU-E-derived erythroblasts was measured with optical sectioning microscopy (9). There were no signifcant $\mathrm{Ca}^{2+}$ gradients between the cytosolic and nuclear compartments in unstimulated human erythroblasts. By contrast, $\left[\mathrm{Ca}_{n}\right]$ was two- to threefold higher than cytosolic free $\mathrm{Ca}^{2+}$ concentration $\left[\mathrm{Ca}_{c}\right]$ in Epo-stimulated cells. Our observation suggests that increases in nuclear $\mathrm{Ca}^{2+}$ may play an important role in gene transcription and cell differentiation.

\section{Methods}

Measurement of empirical three-dimensional point spread function of fluorescence microscopy coupled digital video imaging system. The description and assembly of the various components (listed in legend to Fig. 1) of our fluorescence microscopy coupled digital video imaging system have been detailed previously $(3,4,10)$. In particular, under our experimental conditions used for measuring fluorescence of intracellular fura-2, the intensified charge coupled device (CCD) video camera was always operating in the linear response region (10). In the current study, the only modification to the imaging system was the attachment of a high resolution stepper motor (Unidex 1-A; Aerotech Pittsburgh, PA) to the fine focus adjustment knob of the Zeiss IM 35 inverted microscope.

To reconstruct three-dimensional (3-D) cell image from a stack of two-dimensional (2-D) images, each at slightly different focal planes, it is necessary to have accurate knowledge of the 3-D point spread function (PSF) of the imaging system so that out-of-focus information can be removed from each plane. Since it is difficult to theoretically derive the 3-D PSF of the composite imaging system (objective, microscope relay optics, intensified CCD video camera, digitizing/display board, and high resolution analog monitor), we have chosen to determine the 3-D PSF empirically. An illuminated $0.2 \mu \mathrm{m}$ fluoresbrite-fluorescent monodisperse carboxylated microsphere (excitation $468 \mathrm{~nm}$, emission $532 \mathrm{~nm}$; Polysciences, Inc., Warrington, PA) served as the point source. Since the size of the bead is much smaller than the system 
resolution limit ( $1.0 \mu \mathrm{m}$; reference 10$)$, it effectively behaves as a point object. Digitized images of the fluorescent bead were obtained at 31 focal planes, each differing by $0.25 \mu \mathrm{m}$ in the $z$-axis (Fig. 1). In all experiments, planes were serially digitized by stepwise movement of the objective, and hence focal plane, in the upward direction in order to minimize effects of hysteresis in the focusing mechanism. The empirical PSFs derived from different beads were similar, i.e., images at a given section above or below the most in-focus plane have the same configuration. This observation suggests that the precision of the stepper motor-focussing mechanism is adequate for the present work.

Measurement of intracellular calcium changes in erythroid precursors in response to growth factors. BFU-Es were partially purified from adult peripheral blood and cultured in $0.9 \%$ methylcellulose media as described previously (4). BFU-E derived erythroblasts were removed from culture on day 10 and $\sim 100$ colonies were plucked individually. The cells were pooled, labeled with plateau concentrations of antihuman $\beta 2$-microglobulin which recognizes nucleated cells, and bound to anti-mouse immunoglobulin-coated glass coverslips $(3,4)$. Adherent cells were exposed to fura- 2 acetoxymethyl ester $(2 \mu \mathrm{M} ; 20 \mathrm{~min}$; $37^{\circ} \mathrm{C}$ ), after which they were washed with phosphate-buffered saline (PBS) containing $0.7 \mathrm{mM}$ extracellular $\mathrm{Ca}^{2+}$. Cells loaded with fura-2 were mounted in a Dvorak-Stotler chamber (Nicholson Instruments, Gaithersburg, MD) containing $250 \mu \mathrm{l}$ PBS, and placed on the Zeiss IM 35 microscope. Digitized ( 8 bits per pixel) fluorescent cell images (505 $\pm 17 \mathrm{~nm}$; Ditric Optics, Hudson, MA) at the most in-focus plane were obtained at two excitation wavelengths (350 and $380 \mathrm{~nm} ; 9 \mathrm{~nm}$ bandpass) and stored in a peripheral hard disk drive (Bernoulli Box; Iomega, Roy, UT). Recombinant human erythropoietin $(2 \mathrm{U} / \mathrm{ml}$; AmGen, Thousand Oaks, CA), granulocyte-macrophage colony stimulating factor (GM-CSF, $25 \mathrm{ng} / \mathrm{ml}$; gift of Dr. Steven Clark, Genetics Institute, Cambridge, MA), insulin ( $25 \mathrm{mU} / \mathrm{ml}$ Iletin; Eli Lily and Co., Indianapolis, IN), or Iscove's modified Dulbecco's media (IMDM) (control media used as a dilution buffer for the growth factors) was then added to the same cell and fluorescent cell images were obtained at $1-, 3-$, or 5 -min intervals for $18 \mathrm{~min}$. [Ca $\left.\mathrm{Ca}_{i}\right]$ values of individual cells were derived from the ratiometric images as described previously $(3,4$, 10). Peak $\left[C a_{i}\right]$ responses are shown in Table $I$.

Measurement of subcellular calcium gradients in erythroid precursors. After placement of fura-2 loaded, day 10 erythroblasts on the microscope, paired ( 350 and $380 \mathrm{~nm}$ excitation) fluorescent images at 12 different focal planes $(0.5 \mu \mathrm{m}$ apart) were obtained and stored. Epo ( $2 \mathrm{U} / \mathrm{ml}$ ) was then added to the same cell and a stack of digitized

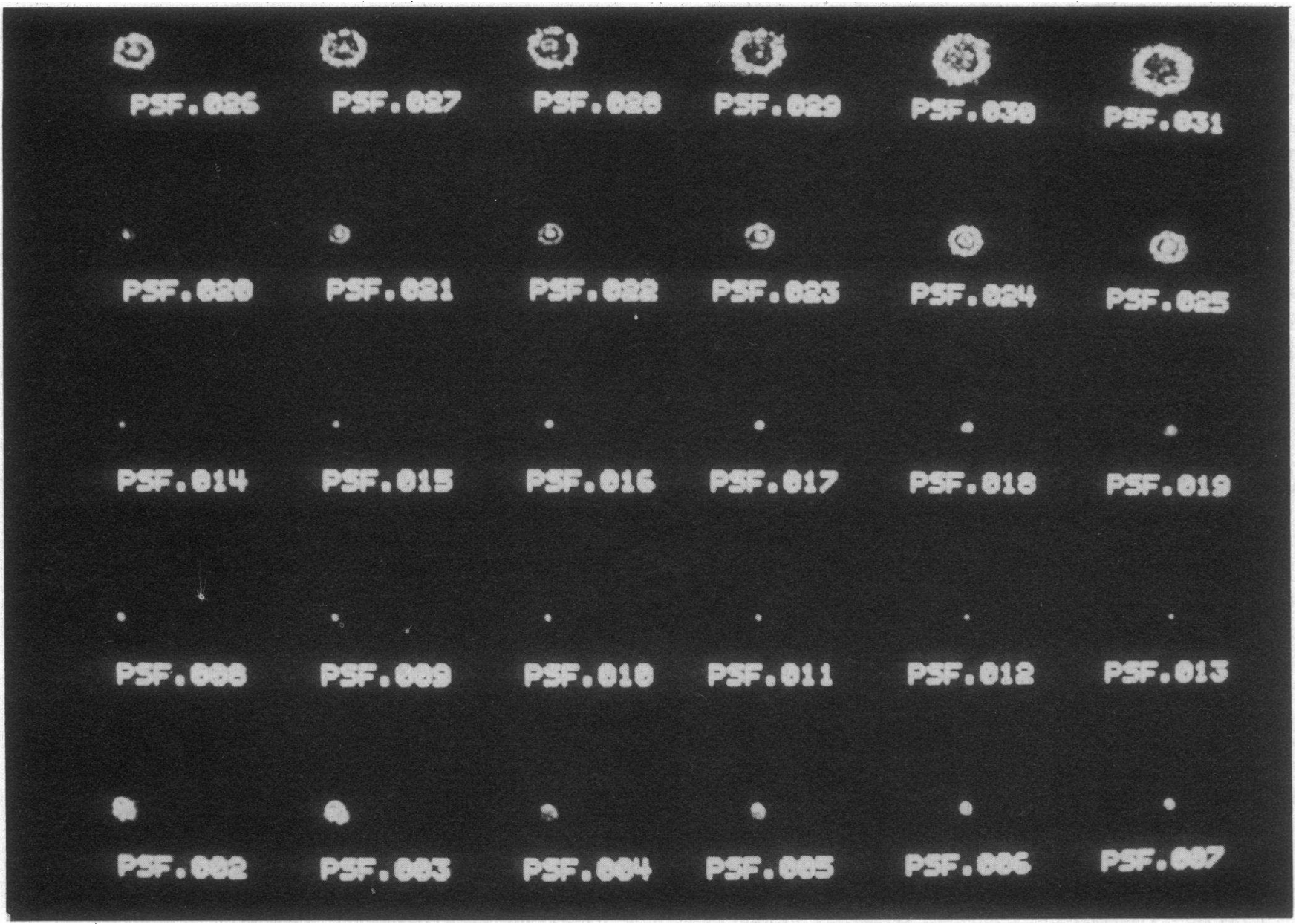

Figure 1. Empirical 3-D point spread function of video imaging system. The front end of the imaging system consists of a Zeiss IM 35 inverted microscope fitted with Zeiss Neofluar 63×/1.25 NA objective and a computer-controlled high resolution stepper motor (Aerotech). Light detector is a Fairchild $3000 \mathrm{~F}$ CCD video camera optically coupled to a Varo microchannel plate image intensifier. Analog output from the camera is fed to an EPIX SVPC-GRB-1MB 8-bit digitizing/display video board residing in IBM PC/AT computer. Images are displayed on Sony PVM $1271 \mathrm{Q}$ high resolution RGB monitor. Serial images of fluorescent microspheres $(0.2 \mu \mathrm{m}$ diam $)$ were captured by stepping the microscope focus by $0.25-\mu \mathrm{m}$ intervals. The numbers represent sections as one approach from the top to the bottom of the microsphere. Plane 13 (PSF 013 ) is the most in-focus view of the microsphere. 
Table I. Peak Intracellular Calcium Responses of Day 10 Erythroblasts

\begin{tabular}{lrcc}
\hline & & \multicolumn{2}{c}{$\left[\mathrm{Ca}_{\mathrm{i}}\right] n M$} \\
\cline { 3 - 4 } & & Baseline & Peak \\
\hline EPO & $(28)$ & $55 \pm 5$ & $225 \pm 39^{*}$ \\
GM-CSF & $(9)$ & $47 \pm 6$ & $85 \pm 11^{*}$ \\
Insulin & $(5)$ & $41 \pm 8$ & $45 \pm 8$ \\
IMDM & $(4)$ & $42 \pm 4$ & $61 \pm 4$
\end{tabular}

Values are means $\pm S E$; numbers in parentheses are numbers of observations. Fura-2 loaded day 10 BFU-E-derived erythroblasts incubated in PBS with $\mathrm{Ca}^{2+}(0.7 \mathrm{mM})$ were exposed to recombinant erythropoietin (EPO, $2 \mathrm{U} / \mathrm{ml}$ ), granulocyte-macrophage colony-stimulating factor (GM-CSF, $25 \mathrm{ng} / \mathrm{ml})$, insulin $(25 \mathrm{mU} / \mathrm{ml})$, or Iscove's modified Dulbecco's medium (IMDM; carrier for EPO, GM-CSF and insulin). Doses of growth factors were chosen to achieve maxi$\mathrm{mal}\left[\mathrm{Ca}_{\mathrm{i}}\right]$ response in BFU-E-derived erythroblasts as determined previously $(3,4)$. Intracellular calcium concentrations $\left(\left[\mathrm{Ca}_{i}\right]\right)$ in each cell were followed at 1,3 , or 5-min intervals for $18 \mathrm{~min}$. There was no difference in baseline $\left[\mathrm{Ca}_{i}\right]$ by one-way analysis of variance. ${ }^{*} P$ $<0.001$ by one-way analysis of variance.

fluorescent cell images at exactly the same focal planes was obtained 15-18 min after Epo addition. Digitized data were transferred to VAX $11 / 780$ computer system for further processing and analysis.

In general, 16 to 32 digitized non-interlaced video frames $(16.7 \mathrm{~ms}$ each) at each excitation wavelength were sequentially added and then separately stored as two 16-bit per pixel accumulated frames. This improves the signal-to-noise ratio of the averaged frame by $\sqrt{16}$ to $\sqrt{32}$. The time required to acquire and accumulate 12 sets of paired 350 and $380 \mathrm{~nm}$ fluorescent images was $\sim 120 \mathrm{~s}$ (including the time to slew the stepper motor 11 times). Since the amplitude of intracellular $\mathrm{Ca}^{2+}$ changes in response to Epo stimulation remained stable $9 \mathrm{~min}$ after Epo addition and persisted for at least 9 additional min (4), the data presented can be considered as steady-state $\left[\mathrm{Ca}_{\mathrm{i}}\right]$ under basal and Epostimulated conditions.

Image analysis. According to the theory of incoherent image formation, the observed image $o_{j}$, for any plane $j$ is a composite of the "true" density distribution $i_{j}$, for that plane with the blurred contributions from the $m$ planes above and the $m^{\prime}$ planes below (11):

$o_{j}=i_{j} * s_{o}+\sum_{\substack{k=j-m^{\prime} \\ k \neq j}}^{j+m} i_{k} * s[(k-j) \cdot \Delta z]$

where $*$ represents the 2-D convolution operation; $o_{j}$ and $i_{j}$ are 2-D functions of the planar coordinates $(x, y) ; s$ is the PSF at each plane of defocus, and $\Delta z$ is the separation between planes. Taking Fourier transforms, the convolution operations in Eq. 1 are converted into multiplications:

$O_{j}=I_{j} \cdot S_{o}+\sum_{\substack{k=j-m^{\prime} \\ k \neq j}}^{j+m} I_{k} \cdot S[(k-j) \cdot \Delta z]$

where $O, I$, and $S$ are the Fourier transforms of $o, i$, and $s$, respectively. Rearranging Eq. 2:

$I_{j}=\left(O_{j}-\sum_{\substack{k=j-m^{\prime} \\ k \neq j}}^{j+m} I_{k} \cdot S[(k-j) \cdot \Delta z]\right) / S_{o}$

Eq. 3 states that the true density distribution at plane $j$ can be obtained by subtracting the blurred contributions from the observed values of the neighboring planes. Eq. 3 can be solved by linear filtering approach, by method of iterative constrained deconvolution, or by sim- ple real and reciprocal space methods as discussed by Agard (9). To simplify computational task, we chose the nearest neighboring approach (12), which is a simplification of the simple real and reciprocal space methods. This is reasonably accurate when using high numerical aperture lenses with relatively large interplanar spacing (9).

Using the nearest neighboring approach $(12,13)$, only the immediately adjacent planes must be considered ( $m=m^{\prime}=1$ in Eq. 3). Since $I_{k}$ is unknown, as a first approximation $O_{k}$ can be taken as $I_{k}$ and Eq. 3 can be simplified to the form:

$I_{j}=C_{1}\left[O_{j}-C_{2}\left(O_{j-1} \cdot S_{1}+O_{j+1} \cdot S_{-1}\right)\right] / S_{o}$

where $C_{1}$ and $C_{2}$ are empirical constants used to balance out the relative contributions of focal plane and adjacent planes. Eq. 4 states that only three sectional planes together with the empirical 3-D PSF are required to restore the in-focus image. Gruenbaum et al. (14) have successfully applied this technique to elucidate the spatial organization of the Drosophila nucleus.

In practice, three adjacent planes $(0.5 \mu \mathrm{m}$ apart $)$ of 350 and $380 \mathrm{~nm}$ fluorescent image data together with their respective 2-D PSFs were fed into VAX 11/780 computer. Background subtraction, Fourier transformation, multiplication of $O_{j}$ 's with their respective contrast transfer functions (CTF, Fourier transform of PSF), and subtraction of blurred contributions from adjacent planes were performed for each of the 350 and $380 \mathrm{~nm}$ images separately. $\mathrm{C}_{2}$ was determined to be 0.32 for 0.5 $\mu \mathrm{m}$ sections (Fig. 2). In general, signals from adjacent planes contributed $\sim 70 \%$ of the total intensity for both 350 and $380 \mathrm{~nm}$ images. It was not necessary to determine $C_{1}$ since by taking the ratio of 350 and $380 \mathrm{~nm}$ image to arrive at calcium concentration, $C_{1}$ cancels out. The resultant images were then processed with the Wiener filter (15) to remove in-focus point spread. The constant for the Wiener filter corresponds to the empirical signal-to-noise ratio of our composite digital video imaging system and was determined by measuring the standard deviation of pixel intensity values of uniform intensity images of free fura-2 solution (16). Following inverse Fourier transform, the restored, in-focus and ratioed $(350 \mathrm{~nm} / 380 \mathrm{~nm})$ images were then processed with a $5 \times 5$ matrix mean filter to limit $x-y$ resolution to $1 \mu \mathrm{m}$ (system resolution limit; reference 10 ) as well as reduce spatial noise and enhance visual presentation. The final processed images were displayed on high resolution RGB monitor (Sony PVM 1271Q), using a pseudocolor (rainbow pattern) code such that red represented the lowest and purple the highest pixel intensity. Pictures of processed images were taken with a conventional $35 \mathrm{~mm}$ SLR camera (Topcon RE super) on Kodak color film (ASA 100).

Calibration of fura-2 signals. The fluorescence intensity ratio $\mathbf{R}$, obtained by division of background subtracted fura- 2 emission (505

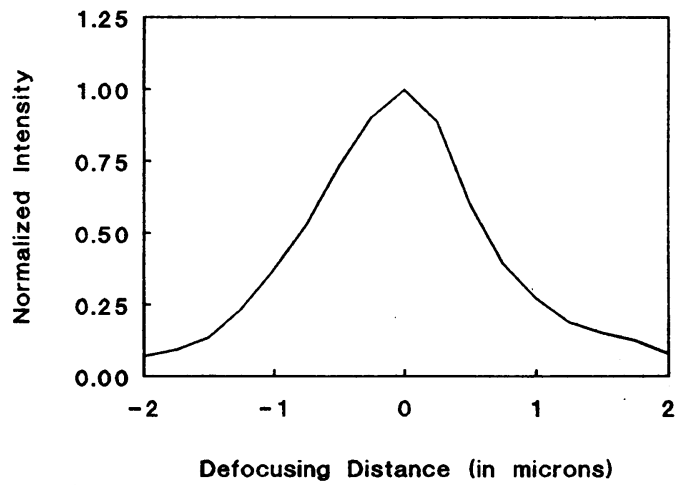

Figure 2. Intensity fall-off profile of point source. The mean pixel intensity (256 gray scale) of the point image at plane 13 (PSF 013; Fig. 1 ) is taken as $100 \%$. Mean pixel intensities of images above and below plane 13 were determined and expressed as a percentage of that of plane 13. From this intensity fall-off profile the constant $C_{2}$ in Eq. 4 (Methods) can be determined to be 0.32 for $0.5-\mu \mathrm{m}$ sections. 
$\mathrm{nm}$ ) at excitation 340 to $350 \mathrm{~nm}$ by that at excitation $380 \mathrm{~nm}$, is related to the free $\mathrm{Ca}^{2+}$ concentration [Ca] (17). To obtain estimates of free $\mathrm{Ca}^{2+}$ concentration from $\mathrm{R}$, an in vitro calibration curve of fura- 2 vs [Ca] was used $(4,10)$. We (4) have previously shown that the dynamic range of intracellular fura-2 was similar to that of free fura- 2 in solution and that $\left[\mathrm{Ca}_{\mathrm{i}}\right]$ in BFU-E derived erythroblasts obtained by in vitro calibration were similar to those derived by in vivo calibration method of Scanlon et al. (18). These observations indicate that in vitro calibration method is valid for determination of $\left[\mathrm{Ca}_{i}\right]$ in human erythroblasts and that incomplete fura-2 acetoxymethyl ester hydrolysis (18) is not an important problem in the present study. In addition, monitoring at the $\mathrm{Ca}^{2+}$-insensitive excitation wavelength $(360 \mathrm{~nm})$ did not show any appreciable loss in $510 \mathrm{~nm}$ epifluorescence from intracellular fura-2 during the 20-min experimental period. This observation suggests that photobleaching of fura-2 (19) did not occur to any significant extent during data acquisition.

\section{Results}

Effects of growth factors on $\left[\mathrm{Ca}_{i}\right]$ in day 10 human $\mathrm{BFU}-\mathrm{E}$ derived erythroblasts. We (4) and others (5) have previously demonstrated that the $\left[\mathrm{Ca}_{i}\right]$ increase in response to Epo stimulation occurred in later (day 10) but not in earlier (day 7) erythroid precursors. Table I shows that in the present series of experiments, Epo induced a $310 \%$ increase in $\left[\mathrm{Ca}_{i}\right]$ while another hematopoietic growth factor GM-CSF had a much more modest effect (80\% increase). Control experiments using IMDM (carrier) or other nonhematopoietic growth factor such as insulin did not result in any appreciable increase in $\left[\mathrm{Ca}_{\mathrm{i}}\right]$ (Table I), suggesting that $\left[\mathrm{Ca}_{\mathrm{i}}\right]$ increase is a relatively specific response to hematopoietic growth factors stimulation in later erythroid precursors. The relatively small $\left[\mathrm{Ca}_{\mathrm{i}}\right]$ increase in response to GM-CSF when compared with the Epo-induced response was also observed previously in erythroid precursors derived from cord blood at similar stages of differentiation (139\% increase for GM-CSF vs. $310 \%$ increase for Epo; reference 3). On account of the small magnitude of $\left[\mathrm{Ca}_{i}\right]$ increase in response to GM-CSF, it was technically difficult to detect changes in subcellular $\mathrm{Ca}^{2+}$ profiles in GM-CSF stimulated cells. Therefore, in this study we concentrate on delineating changes in nuclear to cytosolic $\mathrm{Ca}^{2+}$ gradients in Epo-stimulated erythroid precursors.

Empirical 3-D point spread function of the imaging system. Fig. 1 shows the empirical 3-D PSF of our composite video imaging system, using Zeiss Neofluar $63 \times / 1.25$ NA oil immersion objective. Plane 13 (PSF 013, Fig. 1) is the in-focus image of the point source. Planar images with numbers less than 13 represent images above the focal plane:the lower the number, the higher above the focal plane. Conversely, planar images with numbers higher than 13 are images obtained below the focal plane:the higher the number, the further the distance below the focal plane. Analysis of the point images shows that the images are in the forms of a series of Airy rings that are asymmetrical about the focal plane. Close inspection of the Airy rings indicates that they are also not circularly symmetric. Asymmetry of Airy rings was observed despite imaging different beads, suggesting that our optical system does not exhibit the ideal behavior predicted by diffraction optic theories (20). The nonideal behavior of a microscope video imaging system has been reported previously (21). As demonstrated by Hiraoka et al. (21), the asymmetry of the images above and below the focal plane could be corrected by increasing or decreasing the refractive index of the immersion oil ( $N_{\mathrm{D}} 1.515$ was used in the present experiments) or by the use of objective lenses that have a correction collar to change the optical path length. The lack of circular asymmetry of the Airy rings is most likely due to residual spherical aberrations (22).

From the 2-D PSF images obtained at different focal planes, the corresponding 2-D CTFs were calculated by 2-D Fourier transformation. These were stored in the VAX 11/780 computer system and used in deconvolution operations.

Image intensity of the point source decreased as one traverses above and below the focal plane (Fig. 2). For example, at $1 \mu \mathrm{m}$ above or below the focal plane, image intensity was only $32 \%$ of that at the focal plane. The image intensity fall-off profile was employed to determine the constant $C_{2}(0.32)$ in Eq. 4 used in deconvolution operation.

Improvement in microscopic images by deconvolution. As discussed above, a 2-D microscopic image is not an exact representation of the object but instead is distorted by the optical system. To evaluate the effectiveness of image restoration by the nearest neighboring approach algorithm, we constructed the following theoretical model. A 2-D point source, $P$, was represented by a $2 \times 2$ central region. Identical point sources were positioned directly above and below $P$ at $0.25 \mu \mathrm{m}$ intervals (theoretical depth-of-field) for a distance of $2 \mu \mathrm{m}$ in each $z$-direction. The point sources were convolved with their respective out-of-focus empirical PSFs (Fig. 1) and projected on $P$, which was itself convolved with the in-focus PSF. The resultant composite image is shown in Fig. $3 A$ and represents the "raw, unprocessed image" of $P$ contaminated by light emitting from adjacent planes. Application of nearest neighboring approach deconvolution algorithm (Methods) to the unprocessed image resulted in significant improvement in restoration of the image of point source $P$ (Fig. $3 B$ ). A distortion index, defined by the ratio of number of pixels with positive, non-zero values in the image to the number of pixels occupied by the theoretical point source, is used to quantitate the effectiveness of our deconvolution algorithm. The lower the distortion index, the better is the deconvolution algorithm; 1.0 being the perfect score. The distortion index of the unprocessed image (Fig. $3 A$ ) is 90.3 which improves to 9.3 with nearest neighboring approach deconvolution (Fig. $3 B$ ).
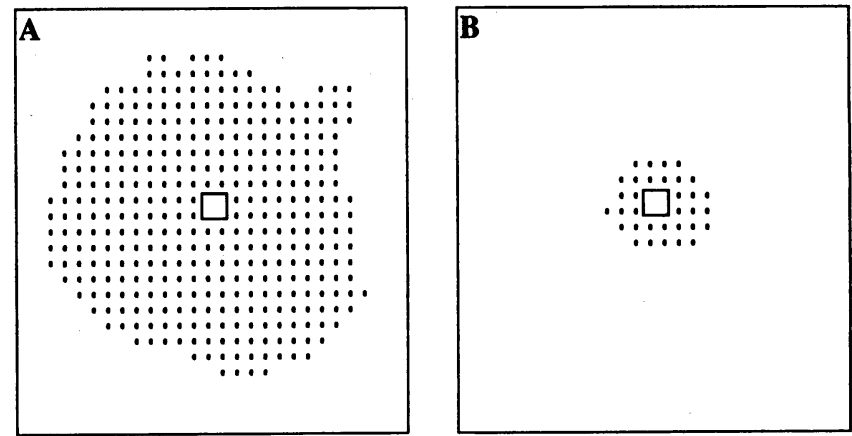

Figure 3. Image restoration by nearest neighboring approach deconvolution algorithm. $(A)$ The image degradation of a theoretical $2 \times 2$ point source by contaminating light arising from adjacent planes (Results). The theoretical point source in the center is represented by the enclosed square while pixels with positive, non-zero intensities are represented by ( . (B) The restoration of the image back towards the point source by nearest neighboring approach. 
To evaluate the numerical accuracy of [Ca] estimates obtained by the nearest neighboring approach deconvolution algorithm, a model cell having two homogeneous compartments (nucleus and cytoplasm) with sharply defined boundaries and spherical symmetry was used to simulate the human erythroblast (Table II). Image formation by the composite video imaging system (mathematically by convolving the model cell with the empirical 3-D PSF) resulted in smearing of fluorescence across boundaries between adjacent compartments. Thus nuclear fluorescence spreads into the cytoplasm and cytosolic fluorescence spreads into the nucleus as well as beyond the cell boundary in both 350 and $380 \mathrm{~nm}$ images of the model cell. In addition, the pixel intensities in a given planar image are much higher than the corresponding ones in the model cell at the same plane, reflecting contributions of contaminating light signals arising from out-of-focus planes. Thus estimates of fluorescence intensities in single wavelength excitation (350 $\mathrm{nm}$ or $380 \mathrm{~nm}$ ) images are gross overestimates (Table II). Processing the "raw" 350 and $380 \mathrm{~nm}$ images with nearest neighboring approach deconvolution algorithm resulted in nearly 19-fold reduction in errors in the estimation of fluorescence intensities in single wavelength excitation images (Table II). In addition, the boundaries between adjacent compartments are more sharply demarcated in the processed images as evidenced by the reduction of noise outside the boundary of the model cell.

Since [Ca] estimates are usually derived from ratiometric $(350 \mathrm{~nm} / 380 \mathrm{~nm})$ but not single excitation wavelength images, we calculated the errors in fluorescence intensities estimated from the raw ratiometric image, i.e., unprocessed $350 \mathrm{~nm}$ image divided by unprocessed $380 \mathrm{~nm}$ image, taken at the plane at cell center. As indicated in Table II, errors in fluorescence intensities measurements (and hence [Ca] estimates) from raw ratiometric images are quite small, although the boundaries between adjacent compartments are blurred and not sharply defined. The small errors in fluorescence intensities estimates from raw ratiometric image is due to the fact that the magnitudes of the contaminating light signals from adjacent planes are similar between the raw 350- and 380-nm images (Table II). Thus for our special case of a model cell with two homogeneous compartments, the raw ratiometric image taken at cell center plane gives a fairly accurate [Ca] estimate of the whole cell $(3,4)$. We wish to emphasize that this conclusion is not valid if heterogeneity exists in [Ca] either in the nuclear or cytosolic compartments or both, or if the plane of the image is not at cell center but rather at the boundary between nucleus and cytoplasm (e.g., at top or bottom of the cell). The processed ratiometric image gives slightly better [Ca] estimates (1.5- to 2-fold reduction in error) in cytosol as well as the whole cell (Table II). More importantly, the boundaries between adjacent compartments are more sharply defined in the processed ratiometric image.

Fig. 4, $A, C$, and $E$ show the unprocessed $350 \mathrm{~nm}, 380 \mathrm{~nm}$ (excitation) and ratioed (350/380) images, respectively, of an erythropoietin-treated human erythroblast taken at cell center. It can be appreciated that the fluorescent cell images appear blurred and fine detail is lost. To remove contaminating light originating from above and below the focal plane, the observed images were subjected to the deconvolution operation using the nearest neighboring approach. Fig. $4, B, D$, and $F$ show the corresponding processed 350-, 380-nm, and ratioed images. Compared with their unprocessed counterparts, the processed images show a marked improvement in image sharpness and detail.

3-D intracellular $\mathrm{Ca}^{2+}$ gradients. Fig. 4, A, B, C, and $D$ show that fura-2 fluorescence signals are generally higher in the nucleus than the surrounding cytoplasm. This may be due to higher [Ca] in the nuclear region, higher free fura- 2 concentration in the nucleus, or less efficient hydrolysis of fura-2 acetoxymethyl ester in the cytoplasm resulting in generation of $\mathrm{Ca}^{2+}$-insensitive fura-2 forms (18). The processed ratioed image (Fig. $4 F$ ) suggests that nuclear [Ca] may indeed by higher than cytoplasmic [Ca] (red rim in Fig. $4 F$ ) since the ratioed image is independent of free fura- 2 concentrations (3,

Table II. Numerical Accuracy of Nearest Neighboring Approach Deconvolution Algorithm

\begin{tabular}{|c|c|c|c|c|c|c|}
\hline \multirow[b]{3}{*}{ Model compartment } & \multicolumn{6}{|c|}{ Error in estimated fluorescence intensity } \\
\hline & \multicolumn{2}{|c|}{$350 \mathrm{~nm}$} & \multicolumn{2}{|c|}{$380 \mathrm{~nm}$} & \multicolumn{2}{|c|}{ Ratiometric } \\
\hline & Imaged model & NNA & Imaged model & NNA & Imaged model & NNA \\
\hline Nucleus & 37.95 & 2.21 & 39.86 & 2.20 & 0.002 & 0.002 \\
\hline Cytosol & 38.76 & 2.25 & 33.32 & 2.27 & 0.010 & 0.005 \\
\hline Total model & 38.05 & 2.21 & 36.55 & 2.23 & 0.003 & 0.002 \\
\hline Outside model & 0.048 & 0.001 & 0.214 & 0.005 & 0.697 & 0.316 \\
\hline
\end{tabular}

A 3-D model cell of the human erythroblast was constructed to have 2 homogeneous compartments with spherical symmetry to represent nucleus ( $80 \%$ total cell diameter) and cytoplasm. To simulate [Ca] determinations from fura-2 fluorescence measurements, both 350-and 380-nm model cells were created. Pixel intensities in the nuclear and cytoplasmic domains in the 350- and 380-nm model cells were set at similar values observed in actual experiments and to reflect the nearly twofold difference in [Ca] between these two regions (Fig. 6). The 350- and 380-nm model cells were convolved with the empirical 3-D PSF (Fig. 1) to simulate the process of image formation and generate the imaged model. The ratiometric imaged model was obtained by division of 350-nm imaged model by the 380-nm imaged model. The unprocessed images at cell center plane of both the 350- and 380-nm imaged models were then subjected to image processing with nearest neighboring approach deconvolution algorithm to generate the processed images (NNA). The processed ratiometric image was obtained by division of the 350-nm processed image by $380 \mathrm{~nm}$ processed image. Errors in estimating fluorescence intensity in a given compartment are estimated as the ratio of $\sum\left(F_{\mathrm{T}}-F_{\mathrm{o}}\right)^{2} / \sum F_{\mathrm{T}}^{2}$, where $F_{\mathrm{T}}$ is the true intensity of a pixel in the model cell and $F_{\mathrm{o}}$ is the observed pixel intensity at the same location in either the unprocessed or processed images (25). Errors calculated for outside the cell model are $\sum F_{\mathrm{o}}{ }^{2} / \sum F_{\mathrm{T}}{ }^{2}$ where $\sum{F_{\mathrm{T}}}^{2}$ is the total squared pixel intensities within the bounds of the model cell (25). 


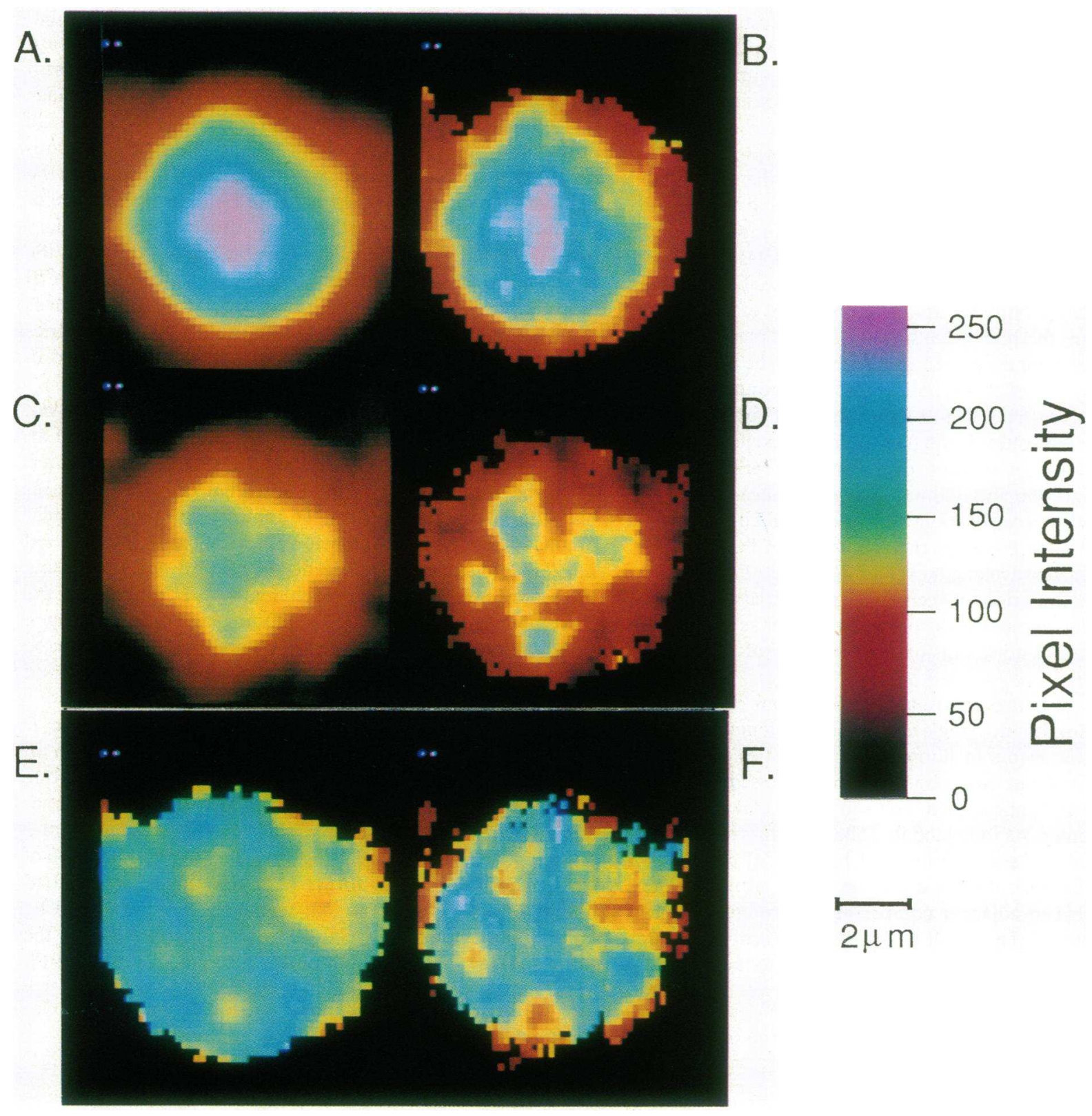

Figure 4. Resolution improvement of 2-D fluorescent cell images. $(A$ and $C$ ) The unprocessed images of intracellular fura-2 fluorescence excited at 350 and $380 \mathrm{~nm}$, respectively. After processing with nearest neighboring approach and using the empirical 3-D PSF, the resultant images are shown in $B(350 \mathrm{~nm})$ and $D(380 \mathrm{~nm})$. Since deconvolution process results in decrease in pixel intensities of processed images by removing light signals from adjacent planes, processed images $(B$ and $D$ ) are multiplied by a factor of 2.75 to bring the pixel intensities to same range of unprocessed images $(A$ and $C$ ) for ease of visual comparison. $(E)$ The ratioed image obtained by division of unprocessed $350 \mathrm{~nm}$ image by unprocessed $380 \mathrm{~nm}$ image. $(F)$ The processed ratioed image. These images are taken at the center plane of an erythropoietin-treated cell and show mostly the nuclear region. A color bar is provided to indicate pixel intensities of a given color.

$4,10,17)$ but reflects free $\mathrm{Ca}^{2+}$ concentrations. To investigate this aspect further, we optically sectioned from the top of the cell to its center. We reasoned that the sections at the top capture more of the cytoplasmic domain, whereas sections at cell center include most of the nuclear region. This is consistent with light microscopic images of day $10 \mathrm{BFU}-\mathrm{E}$ derived human erythroblasts (Fig. 5). Fig. 6, $A, B$, and $C$ show the processed, ratioed images of an erythropoietin-treated cell at 4 , 3 , and $2 \mu \mathrm{m}$ above the center plane, whereas Fig. $6 D$ shows the processed, ratioed image of the same cell taken at center plane.
It can be appreciated that cytoplasmic [Ca] is generally two- to threefold lower than nuclear [Ca]. By assuming that $\mathrm{Ca}^{2+}$ values at cell center plane to be all nuclear and those at $4 \mu \mathrm{m}$ above the center plane to be all cytoplasmic, we calculated $\left[\mathrm{Ca}_{\mathrm{c}}\right]$ of $259 \pm 49 \mathrm{nM}$ and $\left[\mathrm{Ca}_{\mathrm{n}}\right]$ of $434 \pm 24 \mathrm{nM}$ from four cells derived from four different donors. Obviously, these values represent over- and underestimates of $\left[\mathrm{Ca}_{c}\right]$ and $\left[\mathrm{Ca}_{n}\right]$, respectively, in these Epo-stimulated BFU-E-derived erythroblasts.

Although incomplete fura- 2 acetoxymethyl ester hydrolysis in the cytoplasm (17) may theoretically give rise to an arti- 


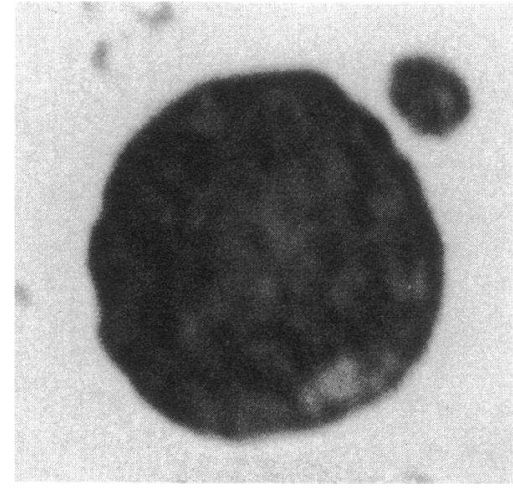

Figure 5. White light image of day 10 BFU-E derived erythroblast. BFU-E derived erythroblasts were plucked from culture on day 10. A cytocentrifuge preparation was made and cells were stained with Wright's stain. Note the thin, dark rim of cytoplasm surrounding the nucleus.

factually higher nuclear [Ca], this is unlikely since the dynamic range of intracellular fura- 2 was similar to that in free solution, suggesting insignificant amounts of intracellular $\mathrm{Ca}^{2+}$-insensitive fura-2 forms (4). To totally eliminate this possibility, we subjected fura- 2 loaded cells to ionomycin treatment in the presence of $4 \mathrm{mM}$ EGTA $\left(R_{\min }\right)$ or $4 \mathrm{mM} \mathrm{CaCl}_{2}\left(R_{\max }\right)(3,4$, $10)$. If the nuclear to cytoplasmic [Ca] gradient is an artifact arising from incomplete fura-2 acetoxymethyl ester hydrolysis in the cytosol, the "gradient" should persist under $R_{\max }$ or $R_{\min }$ conditions. Optically sectioned, processed and ratioed images of erythroblasts taken under $R_{\max }$ and $R_{\min }$ conditions demonstrated that the nuclear to cytoplasmic [Ca] gradient was completely abolished (data not shown). These observations indicate that the nuclear [Ca] gradient cannot be accounted for by incomplete ester hydrolysis.

In contrast to erythropoietin-treated cells (Fig. $6 F$ ), unstimulated human BFU-E-derived erythroblasts did not demonstrate any appreciable nuclear to cytoplasmic [Ca] gradient (Fig. $6 \mathrm{E}$ ). The apparent lack of a nuclear [Ca] gradient in resting cells may be a true phenomenon or may be due to the relatively low intracellular free $\mathrm{Ca}^{2+}$ concentrations (40-50 $\mathrm{nM}$ ) in unstimulated cells (Table I; references 3,4). It is difficult to detect very small regional [Ca] differences in single cells using digital video imaging of fura-2 fluorescence. For example, a change of $10 \mathrm{nM}$ free $\mathrm{Ca}^{2+}$ would only result in a shift of fluorescence intensity ratio ( $R$ ) of fura- 2 solutions by 0.075 (10). Thus at present, we cannot exclude the possibility that a 10-20-nM difference in free $\mathrm{Ca}^{2+}$ concentrations exists between nuclear and cytosolic compartments in resting cells. Likewise, the small increase in $\left[\mathrm{Ca}_{\mathrm{i}}\right]$ over baseline values in response to GM-CSF stimulation (Table I) makes detection of spatial $\mathrm{Ca}^{2+}$ gradients difficult in GM-CSF-stimulated cells using present techniques. In any event, the effects of GM-CSF on nuclear-cytosolic $\mathrm{Ca}^{2+}$ distribution, if any, must be quite modest when compared to those of Epo.

Intracellular $\mathrm{Ca}^{2+}$ values derived from unprocessed ratiometric images at the most in-focus plane $(3,4)$ were quite close to those obtained from processed images at the same plane $(433 \pm 58$ vs. $434 \pm 24 \mathrm{nM})$, at least in the four cells examined in detail by optical sectioning microscopy. The similarity in whole cell [Ca] values between unprocessed and processed ratiometric images at cell center plane is consistent with predictions by our model cell with two compartments, each of which contains homogeneous [Ca] values (Table II). This fortuitous agreement does not detract from the merits of optical sectioning microscopy in that nuclear-to-cytosolic $\mathrm{Ca}^{2+}$ gradients would have been totally masked with conventional $2-\mathrm{D}$ video imaging $(3,4,10)$.

\section{Discussion}

In recent years, intracellular free $\mathrm{Ca}^{2+}$ concentration $\left[\mathrm{Ca}_{\mathrm{i}}\right]$ has been recognized as a regulator of many cellular metabolic processes and as a second messenger of hormones and growth factors (for review, see references 1 and 2). With few exceptions (23-25), most $\left[\mathrm{Ca}_{\mathrm{i}}\right]$ determinations either represent an average of individual values of many cells in the cell suspension $(2,18)$ or the average of regional values within a single cell $(3,4,10)$. The presence of discrete intracellular $\mathrm{Ca}^{2+}$ compartments (endoplasmic reticulum, mitochondria) with $\mathrm{Ca}^{2+}$ buffering capability (26), as well as the gel-like characteristics of the cytoplasm (27) which limits diffusion of ions (28), suggest that ionic gradients may exist within the cell. Such regional ionic gradients cannot be resolved by cell suspension measurements, but are essential to the understanding of subcellular mechanisms of hormone and growth factor action. The introduction of fura-2 (fluorescent $\mathrm{Ca}^{2+}$ indicator with high quantum yield; reference 17), the recent availability of high resolution, solidstate video cameras and electronic hardware for digitizing and storage of individual video frames, and the relative low cost but high speed personal computers, make possible the study of subcellular $\mathrm{Ca}^{2+}$ profiles in real time. Indeed, recent reports have demonstrated the feasibility of measuring spatial $\mathrm{Ca}^{2+}$ gradients in single cells using this approach (23-25).

Perhaps the most important factor for 3-D imaging lies in the selection and proper use of objective lens. The PSF of a lens is a function of both magnification and numerical aperture (NA). In order to reduce the point spread and thereby increase the lateral $(x-y)$ resolution, the use of a high NA lens is preferred. In addition, at low light level emissions characteristic of fluorescence microscopy, the use of a high NA lens has the additional advantage of high light gathering power. High NA objectives with minimal autofluorescence at the working range are especially suited for fluorescence microscopy applications.

The second important factor in data collection is the quantitation of spatial image data. This relates to the selection of a sensitive detector that is used for quantitative measurement of fluorescence emitted by $\mathrm{Ca}^{2+}$-sensitive fluorescent probes in the specimen. We $(10)$ and others $(21,25)$ have shown that the high resolution ( 380 pixels $\times 488$ lines), large dynamic range ( $>1,000: 1)$, photometric linearity, reasonable signal-to-noise ratio ( $>50 \mathrm{db}$ ) and lack of image lag and geometric distortion of a CCD video camera makes it preferable as an imaging device than the more commonly used intensified silicon intensifier target (ISIT) video cameras $(23,29)$. Since fura-2 emissions are of low light intensity, to enhance camera sensitivity and reduce light exposure time (and thus improve temporal resolution), we have elected to couple a microchannel plate image intensifier (Varo, Inc., Garland, TX) to our Fairchild CCD video camera (Fairchild Industrial Products, Winston-Salem, NC). The imposition of the image intensifier in the optical path did not affect the linear response of the imaging device under our experimental conditions, but the intrascene dynamic range and lateral $(x-y)$ resolution were reduced compared to the unintensified $C C D$ video camera (10). 


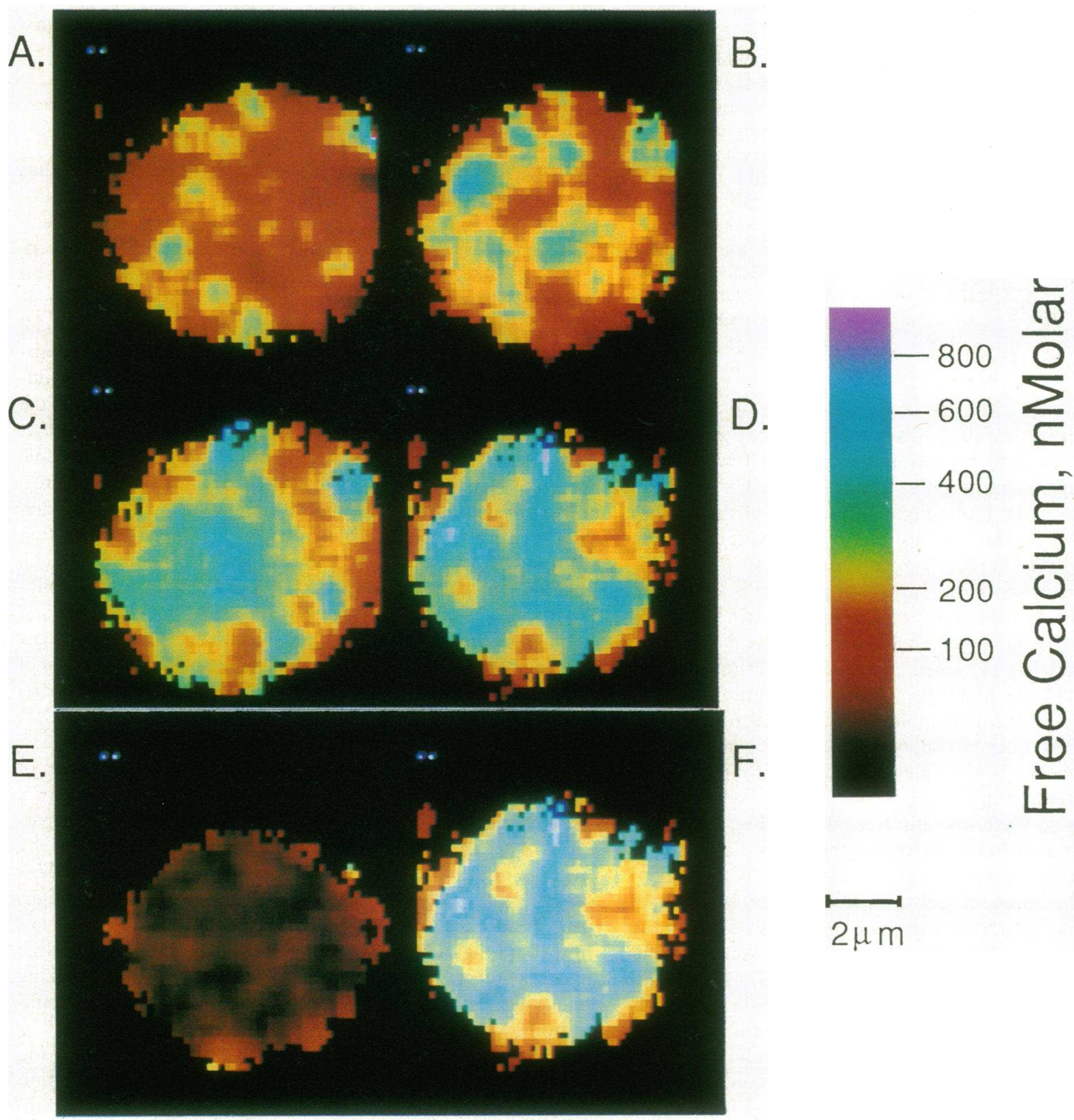

Figure 6. Subcellular calcium gradients in human erythroblasts. $A-C$ The processed ratioed images of an erythropoietin-stimulated erythroblast at 4,3 , and $2 \mu \mathrm{m}$ above the center plane, while $D$ shows the processed ratioed image at the center plane. As one traverses from the top to the center of the cell, one encounters less and less of cytosolic area and more and more nuclear region. The difference in free $\mathrm{Ca}^{2+}$ concentrations between these two compartments is indicated by the color scale bar that was derived from in vitro calibration of fura- 2 fluorescence (Methods and reference 10$).(E$ and $F)$ The processed ratioed images of the same cell before and after erythropoietin stimulation. Both images were taken at the center plane of the cell.

To collect optical section data taken at different focal planes throughout the specimen, it is very important that the exact spacing between adjacent images be accurately and reproducibly controlled. The attachment of a high resolution, computer-controlled stepper motor directly to the fine focusing knob of the microscope is thus desirable in optical sectioning microscopy. With our programmable motion controller system interfaced with IBM PC/AT personal computer through RS-232, the stepper motor assembly has a theoretical resolution of $25 \mathrm{~nm}$ along the optical axis of the microscope that is more than sufficient in our current applications (250 nm steps).

The choice of deconvolution algorithms $(9,25)$ to reconstruct 3-D spatial $\mathrm{Ca}^{2+}$ profiles from a stack of 2-D images depends on several factors. These include desired spatial and temporal resolution, reduction of duration of light exposure to minimize photobleaching of the fluorescent probe (19), and finally, availability of computer time. In theory, the full matrix linear filtering approach (9), the constrained-iterative method (9), and the iterative algorithm utilizing non-negativity con- 
straints and based on regularization theory (25), all of which involve simultaneous manipulation of many sets of data derived from the stack of optical sections, are inherently more accurate and give better spatial resolution than the nearest neighboring approach used in this study. The desire for more detailed spatial information must, however, be balanced by the requirement of temporal resolution of the experimental system as well as the complexity of the computational task. It takes a finite amount of time to capture a sequence of video frames, accumulate multiple video frames into a buffer frame to improve signal-to-noise ratio, transfer the accumulated buffer frame from video board memory to system RAM memory, and slew the stepper motor to the next desired focal plane. Even with our capability to capture alternating 350- and 380$\mathrm{nm}$ frames at video rate (33.3 ms/wavelength change, [10]), it still takes $\sim 8-10 \mathrm{~s}$ to acquire a paired set of 350- and 380-nm buffer frames at one focal plane and to move the objective to the next focal plane. Most of the time spent in data acquisition is incurred during software-directed accumulation of multiple frames into a buffer frame. Since the nearest neighboring approach requires data from only three focal planes, the temporal resolution of our current system is $30 \mathrm{~s}$. This is a significant improvement in temporal resolution over the other more complex deconvolution schemes, which require 10 to 15 sets of data. In addition, nearest neighboring approach has the added advantage in that the computational task is also much less demanding. The faster temporal resolution is especially desirable in tracking relatively rapid hormonally induced $\mathrm{Ca}^{2+}$ transients that usually last only minutes (30). In such situations, the fast biologic response time precludes acquisition of 10-15 sets of data required of the more complex deconvolution algorithms. The nearest neighboring approach allows one to obtain approximate solutions to spatial $\mathrm{Ca}^{2+}$ gradients at one given plane (presumably the most in-focus or most interesting plane) at a relatively rapid sampling rate $(30 \mathrm{~s})$. In the current study involving human BFU-E derived erythroblasts, erythropoietin-induced $\mathrm{Ca}^{2+}$ changes reached steady state after 9-10 min and persisted for many minutes after steady state (3, 4). This fact allowed us time to obtain 12-14 different sections of the cell for 3-D image reconstruction (Fig. 6). Thus, the sole advantage of nearest neighboring approach used in this study is simplification of computational task. We recognize that the nearest neighboring approach is only an approximate method for deconvolution and that subcellular $\mathrm{Ca}^{2+}$ gradients induced by erythropoietin represent steady-state changes rather than rapid $\mathrm{Ca}^{2+}$ transients. In future, the temporal resolution of our imaging system can be improved by reconfiguring the video digitizing/display board such that the frame accumulation process is a hardware feature rather than software-directed.

In single mammalian central nervous system cells, Connor (24) has used an unintensified CCD camera successfully to detect spatial $\mathrm{Ca}^{2+}$ gradients in active growth zones. In contrast to the present study, optical sectioning and subsequent deconvolution were not performed and fine spatial details of subcellular $\mathrm{Ca}^{2+}$ gradients may not be as clearly revealed. In another study of isolated rat cardiac myocytes, Wier et al. (29) did not detect any subcellular heterogeneity in intracellular free $\mathrm{Ca}^{2+}$. While this conclusion is valid from the data presented, small regions of higher $\mathrm{Ca}^{2+}$ (e.g., sarcoplasmic reticulum) (23) may be masked since contaminating light from adjacent planes was not removed by deconvolution. The first study that introduced the concept of deconvolution in the study of subcellular $\mathrm{Ca}^{2+}$ gradients is that by Williams et al. (23). By modeling smooth muscle cells from toad stomach as a perfect cylinder, and knowing the empirical PSF of their imaging system, Williams et al. theoretically calculated what the "true" object should look like from the observed image at one focal plane (23). This modeling effort allowed these authors to conclude that free $\mathrm{Ca}^{2+}$ concentrations in the nucleus and sarcoplasmic reticulum were higher than that in the surrounding cytoplasm. While this simple yet elegant approach is useful in many cell types with well-defined geometry, it is not applicable to all cells. Our study is, to our knowledge, the first to apply optical sectioning microscopy and deconvolution in the study of subcellular $\mathrm{Ca}^{2+}$ profiles. The strategy employed can be applied to all cell types, including cells with varied morphological features not easily modeled by simple geometric patterns.

The major finding in this study is that with erythropoietin stimulation, nuclear $\mathrm{Ca}^{2+}$ was two- to threefold higher than cytoplasmic $\mathrm{Ca}^{2+}$. The existence of a nuclear to cytosolic $\mathrm{Ca}^{2+}$ gradient implies that the nuclear envelope is capable of regulating nuclear $\mathrm{Ca}^{2+}$, as first suggested by Williams et al. (23). Despite the existence of nuclear pores as demonstrated by ultrastructural studies, the pores are not patent but filled with electron-dense material arranged in an orderly manner (31). In addition, protein transport systems have been demonstrated in the nuclear membrane $(32,33)$. In particular, a CaATPase has been reported to exist in nuclear membranes (34). More recently, an ATP-dependent $\mathrm{Ca}^{2+}$ sequestration system has been identified in isolated rat liver nuclei (35). This nuclear $\mathrm{Ca}^{2+}$ transport system operates at submicromolar $\mathrm{Ca}^{2+}$ levels and is distinct from those present in mitochondrial or endoplasmic reticular membranes (35). These observations as well as ours, when taken together, support the notion that $\mathrm{Ca}^{2+}$ permeability barriers and $\mathrm{Ca}^{2+}$ transport systems exist in the nuclear membrane.

Our observation that nuclear free $\mathrm{Ca}^{2+}$ is higher than that in surrounding cytoplasm in erythropoietin-stimulated erythroblasts brings focus to the role of intranuclear $\mathrm{Ca}^{2+} /$ calmodulin-sensitive enzymes (6-8) in cell differentiation and gene transcription. In $\mathrm{GH}_{3} / \mathrm{B}_{6}$ cells, a subclone of the tumor-derived rat pituitary cell line, Laverriere et al. (36) observed that $\mathrm{Ca}^{2+}$ ionophores (ionomycin, A23187) and $\mathrm{Ca}^{2+}$ channel agonist BAY K 8644 enhanced prolactin gene transcription. In murine erythroleukemic cells undergoing differentiation in vitro in response to induction by dimethylsulfoxide or hexamethylene-bis-acetamide, McMahon et al. (37) reported the appearance of a nuclear $\mathrm{Ca}^{2+}, \mathrm{Mg}^{2+}$-dependent endonuclease that was capable of generating single strand DNA breaks in high molecular weight chromosomal DNA. Single strand DNA breaks are known to accompany cell differentiation (37). The observations that $\mathrm{Ca}^{2+}$ ion influx (4), nuclear $\mathrm{Ca}^{2+}$ increase, and $\mathrm{Ca}^{2+}, \mathrm{Mg}^{2+}$-dependent endonuclease activity (37) occurred during a similar early period of erythroid differentiation suggest that these events may be related and involved in the committment of cells to terminal differentiation. Similarly in embryonic chick lens, an endogenous nuclear $\mathrm{Ca}^{2+}, \mathrm{Mg}^{2+}$-dependent endodeoxyribonuclease that was present in both early epithelial (undifferentiated) and late fiber (differentiated) cells remained inactive unless intranuclear concentrations of $\mathrm{Ca}^{2+}$ / $\mathrm{Mg}^{2+}$ were increased (38). In intact isolated rat nuclei, a constitutive endonuclease was activated at submicromolar con- 
centration of $\mathrm{Ca}^{2+}$ with half-maximal activity at $200 \mathrm{nM}$ (39). This level of intranuclear free $\mathrm{Ca}^{2+}$ was certainly achievable in Epo-stimulated erythroblasts. Our current observation, together with evidence cited above, raises the intriguing speculation that with erythropoietin stimulation, cytosolic and intranuclear $\mathrm{Ca}^{2+}$ increase, $\mathrm{Ca}^{2+}, \mathrm{Mg}^{2+}$-dependent endonuclease is activated, chromosomal DNA breakage occurs which then in an as yet unknown manner, commits the cell to terminal differentiation.

In summary, we have demonstrated by optical sectioning microscopy that erythropoietin induces a nuclear to cytosolic $\mathrm{Ca}^{2+}$ gradient in BFU-E derived human erythroblasts. We postulate that the increase in nuclear $\mathrm{Ca}^{2+}$ is related to committing the cell to terminal differentiation, possibly by activating nuclear $\mathrm{Ca}^{2+}, \mathrm{Mg}^{2+}$-dependent endonucleases.

\section{Acknowledgments}

The authors would like to thank Beverly Bell and Tracey Erickson for assistance in the preparation of the manuscript.

This work was supported in part by research grants from the Whitaker Foundation, Juvenile Diabetes Foundation International (187278), the American Heart Association (Pennsylvania Affiliate) (to Dr. Cheung), and in part by Academic Enrichment Fund of the Department of Medicine at the Pennsylvania State University. Dr. Cheung is the recipient of American Heart Association Clinician-Scientist Award 84-429. Dr. Miller is the recipient of the National Institutes of Health (NIH) FIRST Award HL40576 and American Cancer Society Junior Faculty Research Award. Dr. Scaduto, Jr., is the recipient of the NIH FIRST Award DK 40069 and National Kidney Foundation Young Investigator Award. Dr. Tillotson is supported in part by the NIH Research grant DK-40127 and the Whitaker Health Sciences Fund. Dr. Yu is supported in part by U.S. Army Research Office under contract No. DAAL03-87-K-0147.

\section{References}

1. Rasmussen, H., and P. Q. Barrett. 1984. Calcium messenger system: an integrated view. Physiol. Rev. 64:938-984.

2. Moolenaar, W. H., L. H. K. Defize, and S. W. de Laat. 1986. Ionic signaling by growth factor receptors. J. Exp. Biol. 124:359-373.

3. Miller, B. A., R. C. Scaduto, Jr., D. L. Tillotson, J. J. Botti, and J. Y. Cheung. 1988. Erythropoietin stimulates a rise in intracellular free calcium concentration in single early human erythroid precursors. J. Clin. Invest. 82:309-315.

4. Miller, B. A., J. Y. Cheung, D. L. Tillotson, S. M. Hope, and R. C. Scaduto, Jr. 1989. Erythropoietin stimulates a rise in intracellular-free calcium concentration in single BFU-E derived erythroblasts at specific stages of differentiation. Blood. 73:1188-1194.

5. Imagawa, S., B. R. Smith, R. Palmer-Crocker, and H. F. Bunn. 1989. The effect of recombinant erythropoietin on intracellular free calcium in erythropoietin-responsive cells. Blood. 73:1452-1457.

6. Maizels, E. T., and R. A. Jungmann. 1983. $\mathrm{Ca}^{2+}$-calmodulindependent phosphorylation of soluble and nuclear proteins in the rat ovary. Endocrinology. 112:1895-1902.

7. Pardo, J. P., and F. Fernandez. 1982. Effect of calcium and calmodulin on RNA synthesis in isolated nuclei from rat liver cells. FEBS (Fed. Eur. Biochem. Soc.) Lett. 143:157-160.

8. Simmen, R. C. M., B. S. Dunbar, V. Guerriero, J. G. Chafouleas, J. H. Clark, and A. R. Means. 1984. Estrogen stimulates the transient association of calmodulin and myosin light chain kinase with the chicken liver nuclear matrix. J. Cell Biol. 99:588-593.

9. Agard, D. A. 1984. Optical sectioning microscopy: cellular architecture in three dimensions. Annu. Rev. Biophys. Bioeng. 13:191219.
10. Cheung, J. Y., D. L. Tillotson, R. V. Yelamarty, and R. C. Scaduto, Jr. 1989. Cytosolic free calcium concentration in individual cardiac myocytes in primary culture. Am. J. Physiol. 256 (Cell Physiol. 25):C1120-C1130.

11. Yu, F. T. S. 1983. Optical Information Processing. John Wiley and Sons, New York. 157-163.

12. Castleman, K. R. 1979. Digital Image Processing. PrenticeHall, Englewood Cliffs, NJ. 347-379.

13. Weinstein, J., and K. R. Castleman. 1971. Reconstructing 3-D specimens from 2-D section images. Proc. Soc. Photo-Opt. Instrum. Eng. 26:131-138.

14. Gruenbaum, Y., M. Hochstrasser, D. Mathog, H. Saumweber, D. A. Agard, and J. W. Sedat. 1984. Spatial organization of the Drosophila nucleus: A three-dimensional cytogenetic study. J. Cell Sci. 1(Suppl.):223-234.

15. Berriel, L. R., J. Bescos, and A. Santisteban. 1983. Image restoration for a defocused optical system. Appl. Optics. 22:2772-2780.

16. Yelamarty, R. V. 1989. Intracellular calcium gradients in single living cells: Measurement and analysis by optical and digital techniques. Ph.D. Thesis. Department of Electrical Engineering, The Pennsylvania State University, University Park, PA.

17. Grynkiewicz, G., M. Poenie, and R. Y. Tsien. 1985. A new generation of $\mathrm{Ca}^{2+}$ indicators with greatly improved fluorescence properties. J. Biol. Chem. 260:3440-3450.

18. Scanlon, M., D. A. Williams, and F. S. Fay. 1987. A Ca ${ }^{2+}$-insensitive form of fura-2 associated with polymorphonuclear leukocytes. J. Biol. Chem. 262:6308-6312.

19. Becker, P. L., and F. S. Fay. 1987. Photobleaching of fura-2 and its effect on determination of calcium concentrations. Am. J. Physiol. 253 (Cell Physiol. 22):C613-C618.

20. Stokseth, P. A. 1969. Properties of a defocused optical system. J. Opt. Soc. Am. 59:1314-1321.

21. Hiraoka, Y., J. W. Sedat, and D. A. Agard. 1987. The use of a charge-coupled device for quantitative optical microscopy of biological structures. Science (Wash. DC). 238:36-41.

22. Inoue, S. 1986. Video Microscopy. Plenum Press, New York. 113-118.

23. Williams, D. A., K. E. Fogarty, R. Y. Tsien, and F. S. Fay. 1985. Calcium gradients in single smooth muscle cells revealed by the digital imaging microscope using fura-2. Nature (Lond.). 318:558-561.

24. Connor, J. A. 1986. Digital imaging of free calcium changes and of spatial gradients in growing processes in single, mammalian central nervous system cells. Proc. Natl. Acad. Sci. USA 83:6179-6183.

25. Fay, F. S., W. Carrington, and K. E. Fogarty. 1989. Three-dimensional molecular distribution in single cells analysed using the digital imaging microscope. J. Microsc. (Oxf.) 153:133-149.

26. Cheung, J. Y., J. M. Constantine, and J. V. Bonventre. 1986. Regulation of cytosolic free calcium concentration in cultured renal epithelial cells. Am. J. Physiol. 251 (Renal Fluid Electrolyte Physiol. 21):F690-F701.

27. Porter, K. R., and J. B. Tucker. 1981. The ground substance of the living cell. Sci. Am. 244(3):56-67.

28. Nasi, E., and D. L. Tillotson. 1985. The rate of diffusion of $\mathrm{Ca}^{2+}$ and $\mathrm{Ba}^{2+}$ in a nerve cell body. Biophys. J. 47:735-738.

29. Wier, W. G., M. B. Cannell, J. R. Berlin, E. Marban, and W. J. Lederer. 1987. Cellular and subcellular heterogeneity of $\left[\mathrm{Ca}^{2+}\right]_{i}$ in single heart cells revealed by fura-2. Science (Wash. DC). 235:325-328.

30. Bonventre, J. V., K. L. Skorecki, J. I. Kreisberg, and J. Y. Cheung. 1986. Vasopressin increases cytosolic free calcium concentration in glomerular mesangial cells. Am. J. Physiol. 251 (Renal Fluid Electrolyte Physiol. 20):F94-F102.

31. Unwin, P. N. T., and R. A. Milligan. 1982. A large particle associated with the perimeter of the nuclear pore complex. J. Cell Biol. 93:63-75.

32. Dingwade, C., S. V. Sharnick, and R. A. Laskey. 1982. A polypeptide domain that specifies migration of nucleoplasmin into the nucleus. Cell. 30:449-458. 
33. Feldherr, C. M., E. Kallenbach, and N. Salrutz. 1984. Movement of a karyophilic protein through the nuclear pores of oocytes. $J$. Cell Biol. 99:2216-2222.

34. Kulikova, O. G., G. A. Savostianov, L. M. Beliavtseva, and N. I. Razmovskaia. 1982. Issledovanie ATPaznoi aktivnosti i ATP-zavisimoi akkumuliatsii $\mathrm{Ca}^{2+}$ iadrami skeletnykh myshts. Effekty denervatsii i èlektricheskoi stimuliatsii. Biokhimiya. 47:1216-1221. (English Abstr.)

35. Nicotera, P., D. J. McConkey, D. P. Jones, and S. Orrenius. 1989. ATP stimulates $\mathrm{Ca}^{2+}$ uptake and increases the free $\mathrm{Ca}^{2+}$ concentration in isolated rat liver nuclei. Proc. Natl. Acad. Sci. USA 86:453457.

36. Laverriere, J. N., A. Tixier-Vidal, N. Buisson, A. Morin, J. A. Martial, and D. Gourdji. 1988. Preferential role of calcium in the regulation of prolactin gene transcription by thyrotropin-releasing hormone in $\mathrm{GH}_{3}$ pituitary cells. Endocrinol. 122:333-340.

37. McMahon, G., J. L. Alsina, and S. B. Levy. 1984. Induction of a $\mathrm{Ca}^{2+}, \mathrm{Mg}^{2+}$-dependent endonuclease activity during the early stages of murine erythroleukemic cell differentiation. Proc. Natl. Acad. Sci. USA 81:7461-7465.

38. Muel, A. S., E. Chaudun, Y. Courtois, S. P. Modak, and M. F. Counis. 1986. Nuclear endogenous $\mathrm{Ca}^{2+}$-dependent endodeoxyribonuclease in differentiating chick embryonic lens fibers. J. Cell. Physiol. 127:167-174.

39. Jones, D. P., D. J. McConkey, P. Nicotera, and S. Orrenius. 1989. Calcium-activated DNA fragmentation in rat liver nuclei. $J$. Biol. Chem. 264:6398-6403. 